\title{
O IMPACTO DOS INDICADORES SOCIOECONÔMICOS NO GRAU DE EFICÁCIA ORÇAMENTÁRIA
}

\author{
THE IMPACT OF SOCIOECONOMIC INDICATORS \\ ON THE DEGREE OF BUDGET EFFICIENCY
}

\author{
CARLA JANAINA FERREIRA NOBRE \\ Mestra em Ciências Contábeis pela Universidade Federal da Paraíba. \\ Professora substituta da Universidade Federal da Paraíba e \\ professora no Instituto de Educação Superior da Paraíba. Endereço: \\ Universidade Federal da Paraíba / Cidade Universitária - Campus I | \\ Castelo Branco / 58059-900 / João Pessoa/PB / Brasil. \\ E-mail: carlajanainanobre@gmail.com

\section{JOSEDILTON ALVES DINIZ} \\ Doutor em Ciências Contábeis pela Universidade de São Paulo. \\ Professor da Universidade Federal da Paraíba. Endereço: \\ Universidade Federal da Paraíba / Cidade Universitária - Campus I / \\ Castelo Branco / 58059-900 / João Pessoa/PB / Brasil. \\ E-mail: josedilton@gmail.com
}

\section{SEVERINO CESÁRIO DE LIMA}

Doutor em Ciências Contábeis pela Universidade de São Paulo. Professor da Universidade Federal do Rio Grande do Norte. Endereço: Universidade Federal do Rio Grande do Norte / Cidade Universitária - Campus I / Av. Senador Salgado Filho / Natal/RN | Brasil.

E-mail: cesario@ufrnet.br

\section{RONALDO JOSÉ RÊGO DE ARAÚJO}

Mestre em Ciências Contábeis pela Universidade Federal da Paraíba. Professor substituto da Universidade Federal do Rio Grande do Norte. Endereço: Universidade Federal do Rio Grande do Norte / Cidade Universitária - Campus I / Av. Senador Salgado Filho / Natal/RN | Brasil.

E-mail: ronaldocontabilidade@ymail.com

\section{RESUMO}

Estudos têm revelado que a eficácia orçamentária é influenciada pelos indicadores socioeconômicos de uma localidade, tais como renda, saúde, educação e tamanho populacional. Nesse contexto, a eficácia orçamentária é entendida com uma medida que revela se uma organização conseguiu atingir seus objetivos, metas e resultados. Assim, o presente estudo teve como objetivo verificar a relação entre a eficácia orçamentária dos 223 municípios do Estado da Paraíba e as condições socioeconômicas retratadas pelos indicadores sociais e econômicos no período de 2005 a 2013. Como resposta antecipada à questão da pesquisa formulou-se duas hipóteses: $\mathrm{H}_{1}$ : os indicadores socioeconômicos influenciam positivamente a eficácia orçamentária municipal e $\mathrm{H}_{2}$ : os municípios maiores apresentam maior grau de eficácia orçamentária quando comparados com municípios menores. O teste de hipótese foi feito mediante a análise de dados em painel balanceado com efeitos fixos e análise de correlação 
entre as variáveis do modelo. Os resultados revelaram que a eficácia orçamentária municipal é influenciada positivamente pelos indicadores socioeconômicos, confirmando a hipótese 1. A hipótese 2 foi rejeitada. Em suma, o estudo revelou que os indicadores sociais de educação e saúde promovem a eficácia orçamentária dos governos locais.

Palavras-chave: Eficácia orçamentária. Indicadores sociais. Indicadores econômicos.

\begin{abstract}
Studies have shown that budget effectiveness is influenced by the socioeconomic indicators of a locality, such as income, health, education and population size. In this context, budget effectiveness is understood with a measure that shows whether an organization has achieved its objectives, goals and results. Thus, the present study aimed to verify the relationship between the budget effectiveness of the 223 municipalities of the State of Paraiba and the socioeconomic conditions portrayed by the social and economic indicators in the period from 2005 to 2013. As an early answer to the research question two hypotheses were formulated: H1: socioeconomic indicators positively influence municipal budget effectiveness; And H2: the larger municipalities present a greater degree of budgetary efficiency when compared to smaller municipalities. The hypothesis test was performed through balanced panel data analysis with fixed effects and correlation analysis between model variables. The results showed that municipal budget effectiveness is positively influenced by socioeconomic indicators, confirming hypothesis 1. However, hypothesis 2 was rejected. In sum, the study found that social indicators of education and health promote the budgetary effectiveness of local governments.
\end{abstract}

Keywords: Budget efficiency. Social indicators. Economic indicators.

\title{
1 INTRODUÇÃO
}

O planejamento e o controle das atividades realizadas pelas entidades públicas devem ser integrados para que o governo alcance a eficácia na execução do orçamento. Para isso, os gestores precisam pautar suas ações com base nos programas de trabalho fixados no orçamento público, bem como serem essas ações acompanhadas pela sociedade. $O$ orçamento público pode ser definido como um instrumento de previsão de receitas e fixação de despesas. As receitas arrecadadas e as despesas realizadas têm o intuito de garantir continuidade, eficiência, efetividade, eficácia e economicidade dos serviços prestados pelo Estado (Carvalho, 2007). O orçamento é um importante mecanismo de planejamento e controle das atividades realizadas pelas entidades públicas.

Nesse contexto, percebe-se a importância da execução orçamentária apoiada nos instrumentos de planejamento e controle para que a administração pública alcance maior eficácia do orçamento. Castro (2006) relaciona a eficácia como algo que se preocupa com os fins, na intenção de atingimento dos objetivos. Interpretando esse conceito, pode-se entender a eficácia como o alcance dos objetivos e metas que foram planejados no orçamento.

A sociedade é inserida nessa abordagem segundo 0 atendimento das suas necessidades nas áreas de saúde, educação, lazer, segurança, transporte, dentre outras. Então, o orçamento eficaz promove melhorias nos direitos assegurados à comunidade, impactando positivamente os indicadores socioeconômicos de uma localidade.

Os indicadores econômicos e sociais possibilitam avaliar a situação e aprimoramento da sociedade. É essencial à compreensão dos seus significados e limitações (Moldau, 1998). Portanto, estudar esses indicadores sob a perspectiva da eficácia orçamentária pode ser primordial para o entendimento da estrutura de planejamento e execução das ações governamentais.

Os índices sociais e econômicos podem ter reflexos significativos nesse planejamento, visto que a administração pública estará atendendo aos anseios dos cidadãos. Diante disso, tem-se como principais áreas para esta pesquisa indicadores que reflitam realizações na educação, na saúde e na renda da comunidade, acompanhadas por indicadores socioeconômicos. Para Lima e Diniz (2016), os indicadores socioeconômicos apontam, resumidamente, os determinantes das necessidades sociais e econômicas de uma sociedade. 
Esses determinantes podem ser apontados como por exemplo a renda, a saúde, a educação, indicando os pontos em que o governo deve dar maior atenção.

A eficácia orçamentária beneficia dois importantes stakeholders do ambiente governamental: a sociedade e o governo, cada um deles com suas peculiaridades, mas com interesses convergindo para um objetivo comum, a concretização das ações governamentais previstas no orçamento. Segundo Freeman (2010), stakeholders é qualquer indivíduo ou grupo de indivíduos que possa afetar o alcance dos objetivos organizacionais, isto é, um grupo de pessoas interessadas em um mesmo negócio. Assim, os interesses compatíveis e incompatíveis dos stakeholders precisam ser conciliados para que os objetivos organizacionais sejam atingidos.

Nesse sentido, Boaventura, Cardoso, Silva e Silva (2009) assinalam que entre as contribuições da teoria do stakeholders destaca-se a postura administrativa da organização, que se torna mais estratégica e traz benefícios para a entidade. Na visão de Donaldson e Preston (1995), a teoria dos stakeholders está alicerçada em bases éticas. Permite identificar o papel e a importância de cada um dos stakeholders.

Neste cenário, os pilares dessa teoria são fundamentais na área governamental, visto que os interesses dos gestores não devem prevalecer sobre o bem-estar da população. Portanto, a comunidade deve conhecer a atuação do Estado, na qual devem estar contemplados os interesses do cidadão. Então, os cidadãos necessitam ser estimulados a participarem efetivamente da elaboração, acompanhamento e avaliação das políticas públicas (Borges \& Pereira, 2014).

Nesse contexto, é importante destacar que a sociedade tem se tornado mais exigente e mais participativa nas últimas décadas. No campo educacional, conforme afirma Lück (2000), a sociedade está mais exigente por conhecer que a educação constitui grande valor para o desenvolvimento social. Nogueira (2004) afirma que numa democracia participativa é essencial a presença de cidadãos que sejam capazes de colaborar para a melhoria da gestão pública. Para isso, os governantes devem expor decisões e orientações claras, apontar caminhos e possibilidades aos atores sociais.

Assim, para que haja efetivo avanço na eficácia da gestão pública, conforme Litpvsky e MacGillivray (2007), deve haver colaboração mútua entre os atores do desenvolvimento: governantes e governados, distribuindo responsabilidades para que haja resultados substanciais. Nesse sentido, o Brasil tem apresentado melhoria na participação social quanto à cobrança da eficácia e transparência das contas públicas. No entanto, a inclusão dos pobres ainda é um desafio (Litpvsky e Macgillivray, 2007).

Com base nessas abordagens teóricas, levantou-se o seguinte questionamento para investigação: qual a relação entre a eficácia orçamentária dos municípios do Estado da Paraíba e as condições socioeconômicas?

Visando a antecipar uma resposta ao questionamento formulado, foram definidas as seguintes hipóteses: $\mathrm{H}_{1}$ : os indicadores socioeconômicos influenciam positivamente a eficácia orçamentária municipal e $\mathrm{H}_{2}$ : os municípios maiores apresentam maior grau de eficácia orçamentária quando comparados com municípios menores.

Assim, o objetivo principal do presente estudo consistiu em verificar a relação entre a eficácia orçamentária dos municípios do Estado da Paraíba e as condições socioeconômicas retratadas pelos indicadores sociais e econômicos.

A presente pesquisa busca contribuir com conhecimentos sobre a eficácia orçamentária, para a evolução da ciência contábil, bem como auxiliar na gestão municipal. Dessa forma, a pesquisa se justifica pela importância que uma gestão orçamentária coerente traz para o Estado, do ponto de vista do controle social, econômico e organizacional. Apresenta os benefícios que podem vir a refletir nas condições socioeconômicas da população.

Este artigo inicia-se com esta introdução. No segundo tópico apresenta-se o referencial teórico. Em seguida são apresentados os aspectos metodológicos e a análise dos resultados. Finalmente, são apresentadas as considerações finais.

\section{REFERENCIAL TEÓRICO}

Neste referencial teórico são abordados aspectos da eficácia orçamentária, como também a sua relação com os indicadores socioeconômicos e a teoria dos stakeholders. 


\subsection{Eficácia orçamentária}

A concepção de orçamento-programa tem respaldo na Constituição da República Federativa do Brasil (1988), na Lei n. 101 (2000), na Lei n. 4.320 (1964), Decreto-Lei n. 200 (1967). Os fundamentos do orçamento-programa centram-se no elo entre orçamento e planejamento, visando conferir a esse instrumento, além da função de controle, a função de gestão. Isso marca a transição do orçamento tradicional para o moderno. Carvalho (2007) verifica as vantagens da utilização do orçamento-programa. Explana os benefícios ao trabalho do governo, aos seus projetos e atividades, objetivos, metas, custos e resultados, o que gera maior transparência pública.

Conforme Giacomoni (2012), o orçamento-programa foi baseado no orçamento de desempenho experimentado pelos Estados Unidos, e começou a ser disseminado pelo mundo na década de 1950, especialmente por iniciativas da Organização das Nações Unidas (ONU). Passou por modificações na implantação no Brasil. Porém, apenas em 1974, é que o governo brasileiro incluiu a classificação programática pela necessidade de informações sobre as programações do governo e, também, pela integração de planejamento e orçamento (Kashiwakura, 1997).

Dessa forma, entende-se que o orçamento planejado de forma coerente, de modo que os objetivos sejam atingidos, pela integração governo e sociedade, as condições socioeconômicas dos municípios tendem a melhorar, atendendo à eficácia orçamentária. A questão da eficácia está centrada nos produtos que os governos oferecem à comunidade, ou seja, nos outputs. Esses, de acordo com Lima e Diniz (2016), podem ser visualizados como unidades físicas dos bens e serviços que são resultados do processo produtivo, destacando as dificuldades em se identificá-los.

Assim, a eficácia é uma medida em que uma organização consegue atingir seus objetivos, suas metas e outros resultados, ou seja, é a relação entre os resultados que são esperados e os outputs de bens e informações (Controladoria Geral da União [CGU], 2009). A relação entre eficácia e orçamento-programa se encontra na satisfação das necessidades do cidadão no que tange aos bens e serviços públicos, principalmente no enfoque dos resultados, ou seja, se a execução do orçamento-programa atende às necessidades da população (Koscianski, 2003).

Mas, a prestação dos serviços não pode ocorrer de qualquer forma. A qualidade dos serviços deve ser inerente à sua prestação. Com isso, quando no planejamento orçamentário são postas todas as reais necessidades do município e os objetivos traçados são atendidos, pode-se entender que a eficácia orçamentária foi alcançada. Pois, como afirma Bezerra (2013), a eficácia acontece no momento em que os objetivos preestabelecidos são alcançados.

O planejamento orçamentário no Brasil é feito por meio dos instrumentos conhecidos como Plano Plurianual (PPA), Lei de Diretrizes Orçamentárias (LDO) e Lei Orçamentária Anual (LOA), todos previstos na Constituição da República Federativa do Brasil (1988), como também na Lei de Responsabilidade Fiscal (LRF) (2000). Conforme Carvalho (2007), o PPA é o planejamento estratégico de médio prazo; a LDO é o planejamento tático. Representa as diretrizes para elaboração da LOA, isto é, faz a ligação entre o PPA e a LOA; e a LOA representa o planejamento operacional da gestão pública, pois efetiva os objetivos e metas que foram estabelecidos no PPA.

Dessa forma, esses instrumentos de planejamento, alinhados às necessidades sociais, promovem a identificação das prioridades da sociedade que coincidem com as prioridades do governo, quais sejam: garantir maior transparência, integrar o planejamento ao orçamento, equilibrar receitas e despesas, promover uma adequada execução orçamentária, apresentar melhorias na saúde, educação, segurança e assistência social, e, essencialmente, alcançar a eficácia orçamentária.

Todavia, se as dotações planejadas inicialmente na lei orçamentária não forem suficientes ou não forem previstas, o gestor pode legalmente abrir crédito adicional para incluir novas despesas ou reforçar as já existentes (Carvalho, 2007). Tal procedimento poderá prejudicar a eficácia orçamentária caso os recursos de cobertura sejam insuficientes. Nesse sentido, Santana, Pessoa, Cabral, Santos, Diniz (2007) acreditam que a alteração no orçamento dos municípios contrariam o arcabouço teórico do planejamento e orçamento, pois, se o planejamento é a forma de previsão das despesas, que serão realizadas em determinado 
período, os créditos adicionais só seriam necessários em caso de despesa de extrema urgência. Assim, se o gestor não abrir créditos adicionais durante o exercício, a eficácia orçamentária não será tão impactada negativamente.

Portanto, é necessário que o planejamento e a execução orçamentária estejam integrados para que a eficácia seja alcançada, atendendo, assim, às necessidades da população e contribuindo para a melhoria das ações de saúde e educação e alavancando a renda da comunidade do município.

\subsection{Indicadores socioeconômicos e a eficácia orçamentária}

Os indicadores sociais são importantes para se medir o perfil da realidade de um município. Permitam traçar políticas públicas mais acertadas (Jannuzzi, 2012). Lima e Diniz (2016) denotam que esses indicadores são compostos por variáveis sociais e econômicas e medem as necessidades da população. Eles podem ser, por exemplo, a renda, o desemprego, o transporte, os índices de natalidade e mortalidade, os índices de analfabetismo, etc.

Jannuzzi (2002) conceitua indicador social como uma medida quantitativa que teoricamente interessa às pesquisas acadêmicas e de forma programática auxilia na formulação de políticas. Aristigueta, Cooksy e Nelson (2001) argumentam que os Estados veem os indicadores sociais como uma ferramenta fundamental de governança; e ainda que são estratégias para gestão por resultados. Os autores (2001) trazem os indicadores sociais como sinais de saúde social e econômica.

Conforme Jannuzzi (2002), para que esses indicadores estejam presentes em pesquisas acadêmicas é de suma importância que observem uma série de requisitos, tais como, sensibilidade às políticas públicas e desagregação em termos geográficos e históricos, para que haja possíveis comparações no decorrer do tempo. Assim, os diagnósticos socioeconômicos apresentados por esses indicadores tornam-se relevantes, válidos e confiáveis para prover uma contínua alocação de recursos e implementação de políticas públicas.

Diversas pesquisas utilizaram os indicadores socioeconômicos, relacionando-os com a eficácia orçamentária, e, principalmente, associando-os com a transparência pública. Aristigueta et al. (2001) analisaram o papel dos indicadores sociais sobre um sistema de resultados orientado em um estudo de caso de Delaware. Constataram que esses indicadores servem para controlar o desempenho das instituições, como também as medidas de bem-estar das famílias, crianças e comunidades. Dessa forma, fornecem informações sobre a sociedade, suas necessidades e satisfação.

Quanto ao grau de eficácia orçamentária o estudo de Santana et al. (2007) verifica que os municípios paraibanos não foram impactados pela LRF (2000) no período imediatamente anterior e posterior à implantação da lei, porém comparado a anos posteriores verificou-se significância estatística entre a eficácia orçamentária e as despesas fixadas e executadas, ou seja, a LRF influenciou positivamente na melhoria do planejamento público.

Queiroz, Nobre, Silva e Araújo (2013) estudaram o nível de evidenciação de informações em municípios do Rio Grande do Norte, incluindo a quantidade da população. $O$ estudo constatou fraca relação entre o tamanho do município e o índice de evidenciação. Além disso, Queiroz et al. (2013) utilizaram variáveis socioeconômicas. Constataram que municípios que possuem um nível de desenvolvimento socioeconômico mais elevado tendem a divulgar mais informações.

Poker, Nunes e Nunes (2013) podem sustentar o uso de duas variáveis destacadas neste artigo: renda e educação. Em sua pesquisa os autores permitiram concluir que políticas públicas devem desenvolver maior investimento em educação, e para isso utilizou o fator renda em seus testes empíricos. Além disso, Poker et al. (2013) esclarecem que existe ineficiência relativa dos municípios com maior zona rural, o que indica a necessidade de políticas públicas específicas.

Neves, Diniz e Martins (2015) relacionam transparência pública e IDHM, como proxy da condição socioeconômica. Apontam que existe relação entre indicador socioeconômico e nível de transparência pública, ou seja, municípios com melhores indicadores socioeconômicos tendem a apresentar maior transparência da gestão.

Wright e Paulo (2014) sustentam o uso das variáveis renda e educação, que também estão evidenciadas nesta pesquisa. Esses autores estudaram os elementos determinantes da 
transparência fiscal ativa nos municípios brasileiros, comprovando que as variáveis idade, educação e renda apresentaram correlação com a variável transparência.

Oliveira e Passador (2014) fizeram uma análise da utilização da saúde na avaliação da alocação dos recursos dos municípios. Encontraram que municípios que gastam mais recursos em atenção básica têm maior nível de desempenho no sistema. Além disso, constataram que municípios com maior população têm resultados piores em saúde, pois o acesso a esses serviços é prejudicado pela alta demanda.

A maioria das pesquisas feitas utilizando indicadores socioeconômicos compara-os à transparência pública, mas servem para apoiar esta pesquisa, visto que essa dimensão é um pressuposto da eficácia orçamentária.

Dentre os principais indicadores utilizados nesta pesquisa destacam-se os de educação, renda e saúde. Para representar cada um dos indicadores será utilizado o Índice FIRJAN de Desenvolvimento Municipal (IFDM), no nível consolidado, como também, notas discriminadas de Emprego e Renda, Educação e Saúde. Trata-se de um sistema que acompanha de forma anual o desenvolvimento econômico de todos os municípios brasileiros nas três áreas supracitadas.

A gestão pública é auxiliada por esses indicadores, que constatam as demandas e diagnosticam a situação econômica e social da comunidade (Varela, 2004). Portanto, as informações geradas a partir dos indicadores socioeconômicos auxiliam na análise da saúde do governo e das necessidades da sociedade.

\subsection{A teoria dos stakeholders e a eficácia orçamentária}

A teoria dos Stakeholders apareceu pela primeira vez devido à insatisfação surgida pelo fato dos critérios financeiros de eficácia serem unilaterais (Gomes, 2006). A eficácia não pode ser medida apenas por satisfazer a uma das partes interessadas, mas a todos os agentes que participam de uma organização.

Os stakeholders representam as várias partes envolvidas em uma relação de negócio, ou seja, são os indivíduos interessados em determinadas ações. Devido às dificuldades da gestão em identificar cada stakeholder, para que todas as partes interessadas se sintam satisfeitas em qualquer tipo de negociação em que estejam inseridas, os defensores da teoria dos stakeholders, como Donaldson e Preston (1995), identificam a importância dessa teoria nesse contexto.

Com relação à eficácia, a teoria dos stakeholders pode ser destacada como a perspicácia em atender, ou seja, em garantir a satisfação não só dos acionistas, mas de todos os que têm vínculo com a organização. No âmbito do setor público, pode-se resumir os stakeholders em governo e população. Porém, tanto o governo como as pessoas que compõem uma comunidade apresentam necessidades, anseios e desejos diferenciados. Além disso, outros componentes são englobados no contexto governo e sociedade, os quais aumentam a complexidade da gestão dos stakeholders, tais como fornecedores, instituições financeiras e acionistas.

Por isso, a dificuldade em se fazer uma apropriada gestão dos stakeholders. Conforme Bandeira-de-Melo, Marcon e Alberton (2005), a verificação empírica dessa gestão ainda é escassa na literatura. Devido a exigências diferenciadas, o governo deve analisar quais são os aspectos primordiais e comuns para a sociedade.

Os gestores são acometidos por diversas dificuldades para administrar os serviços públicos. Uma forma de observação das necessidades da sociedade é a verificação quantitativa dos indicadores sociais, extraindo as informações necessárias para que haja a melhoria na elaboração do planejamento orçamentário. Essa é uma forma de dialogar com os stakeholders da sociedade, evidenciando um guia das organizações e instituições nas quais atuam, ou seja, mostrando algumas disposições gerais como o uso do espaço, o grau de influência das organizações e as dificuldades rotineiras (Santos, 2008).

No entanto, tendo em vista ser difícil identificar as necessidades da população, os gestores precisam da participação popular, uma vez que se acredita que essa participação tenha maior capacidade de identificar as necessidades dos cidadãos. Isso se faz mais evidente em países democráticos, em que o governo tem expectativas de aumentos e ampliação de programas, tais como saúde, educação e distribuição de renda (Khan \& Hildreth, 2002). 
Com essa análise, pode-se inferir um fortalecimento da relação entre os stakeholders, consolidando o interesse público nas áreas como educação, saúde, segurança e infraestrutura. Governo e sociedade, por meio de interesses mútuos, devem chegar a um consenso das reais necessidades de um município para que a atenção básica seja direcionada para esses fins, fazendo com que os stakeholders mantenham uma relação que beneficie a condição socioeconômica da sociedade, tendendo à eficácia orçamentária.

\section{METODOLOGIA}

Nesta seção são apresentados os procedimentos metodológicos: universo e dados, modelo e hipóteses da pesquisa, definição das variáveis do modelo.

\subsection{Universo e dados da pesquisa}

Para a consecução dos objetivos desta pesquisa foram coletados dados referentes aos 223 municípios paraibanos no período de 2005 a 2013. Em virtude da dificuldade de acesso e pelo motivo desses anos serem os mais atuais com disponibilidades de dados, o universo temporal serviu para constatar os avanços ou retrocessos na execução orçamentária em relação ao aspecto de eficácia e seus impactos nos indicadores socioeconômicos.

Os dados foram coletados nos portais do Instituto Brasileiro de Geografia e Pesquisa [IBGE], na base do SAGRES (Sistema de Acompanhamento da Gestão dos Recursos da Sociedade) do Tribunal de Contas do estado da Paraíba e no portal do índice FIRJAN.

\subsection{Modelo e hipóteses da pesquisa}

Para responder a questão de pesquisa inicialmente formulada, foi desenvolvido um modelo de regressão segundo a Equação a seguir.

Em que:

$$
G E O_{t}=\beta_{0}+\beta_{1} S A U D E_{i t}+\beta_{2} E m p R e n d a_{i t}+\beta_{3} E D U C_{i t}+\beta_{4} H A B M_{i t}+\mu
$$

$\beta_{0}$ - é a constante do modelo;

GEO - grau de eficácia orçamentária;

SAUDE - índice FIRJAN de desenvolvimento municipal da saúde;

EmpRenda - emprego e renda;

EDUC - índice FIRJAN de desenvolvimento municipal da educação;

HABM - quantidade de habitantes do município;

$\mu$ - é o termo de erro.

Diante do modelo exposto, o estudo formulou as hipóteses a seguir, no intuito de verificar se os indicadores socioeconômicos são capazes de impactar a eficácia orçamentária nos municípios paraibanos. municipal.

$\mathrm{H}_{1}$ : os indicadores socioeconômicos influenciam positivamente a eficácia orçamentária

Essa hipótese foi formulada tendo em vista que a relação entre os municípios e seus indicadores socioeconômicos traduz uma oportunidade para a observação do comportamento orçamentário diante de questões sociais, como a falta de recursos financeiros (Bárbara \& Rodrigues, 2005).

$\mathrm{H}_{2}$ : os municípios maiores apresentam maior grau de eficácia orçamentária quando comparados com municípios menores.

Essa hipótese foi formulada tendo em vista que em municípios com maior população a arrecadação de tributos tende a ser maior, conforme achados de Mendes, Miranda e Cossio (2008), ao explanarem que um maior desenvolvimento econômico tende a expressar maior capacidade de arrecadação. Além disso, a necessidade de gastos é influenciada por tamanho e crescimento da população. 


\subsection{Definição das variáveis do modelo}

Consoante modelo desenvolvido na Equação 1 acima, o quadro a seguir descreve a variável dependente - grau de eficácia orçamentária - e respectivas variáveis independentes, representadas por variáveis sociais e econômicas.

Tabela 1

Descrição das variáveis utilizadas no modelo da pesquisa

\begin{tabular}{|c|c|c|c|}
\hline VARIÁVEIS & SIGLA & DEFINIÇÃO OPERACIONAL & JUSTIFICATIVA \\
\hline $\begin{array}{l}\text { Grau de } \\
\text { eficácia } \\
\text { orçamentária }\end{array}$ & GEO & $\begin{array}{l}\text { Essa variável será medida com a Equação } \\
2 \text { a seguir, utilizando-se dados extraídos } \\
\text { do portal SAGRES do Tribunal de Contas } \\
\text { do Estado da Paraíba. }\end{array}$ & $\begin{array}{l}\text { A utilização dessa variável está } \\
\text { apoiada nos estudos de Santana et } \\
\text { al. (2007), e serve para diminuir } \\
\text { possíveis inconsistências nas } \\
\text { variações monetárias no decorrer } \\
\text { do tempo; por isso a importância da } \\
\text { medida em índice. O grau de } \\
\text { eficácia ideal é } 1 \text {. Será atribuído } \\
\text { bom grau de eficácia orçamentária } \\
\text { para os municípios que } \\
\text { apresentarem GEO entre } 0,9 \text { a } \\
1,10 \text {. }\end{array}$ \\
\hline $\begin{array}{l}\text { Quantidade de } \\
\text { habitantes do } \\
\text { município }\end{array}$ & HABM & $\begin{array}{l}\text { O número de habitantes foi coletado do } \\
\text { portal do IBGE, com base no censo } 2010 \text { e } \\
\text { nas estimativas dos demais anos } \\
\text { estudados nesta pesquisa (2005, 2006, } \\
2007,2008,2009,2011,2012 \text { e } 2013) \text {. }\end{array}$ & $\begin{array}{l}\text { Acredita-se que municípios maiores } \\
\text { apresentam maior eficácia } \\
\text { orçamentária, conforme sustentado } \\
\text { nos trabalhos de Queiroz et al. } \\
(2013) \text { e Oliveira e Passador } \\
(2014) \text {. }\end{array}$ \\
\hline $\begin{array}{c}\text { Emprego e } \\
\text { Renda }\end{array}$ & EmpRenda & $\begin{array}{l}\text { Para essa variável foi utilizado como proxy } \\
\text { o índice FIRJAN, visto conter os anos a } \\
\text { que este estudo se propõe. A nota para } \\
\text { esse índice é atribuída da seguinte forma: } \\
\text { utilizando a geração de emprego formal e } \\
\text { a capacidade de absorção da mão de obra } \\
\text { local, a geração de renda e sua } \\
\text { distribuição no mercado de trabalho do } \\
\text { município. Foi atribuído peso de } 25 \% \text { em } \\
\text { cada conceito. A fórmula é apresentada } \\
\text { abaixo conforme numeração Equação } 3 \text {. }\end{array}$ & $\begin{array}{l}\text { Pressupõe-se que quanto melhor a } \\
\text { eficácia orçamentária, maior será a } \\
\text { renda dos cidadãos, conforme } \\
\text { sustentam os estudos de Queiroz et } \\
\text { al. (2013), Poker et al. (2013), } \\
\text { Neves et al. (2015) e Wright e Paulo } \\
\text { (2014). }\end{array}$ \\
\hline Educação & EDUC & $\begin{array}{l}\text { Para essa variável foi utilizado como proxy } \\
\text { o índice FIRJAN, visto conter os anos a } \\
\text { que este estudo se propõe. A nota para } \\
\text { este índice é atribuída da seguinte forma, } \\
\text { com os pesos indicados após cada item: } \\
\text { utilizando as matrículas na educação } \\
\text { infantil - } 20 \% \text {; o abandono no ensino } \\
\text { fundamental - } 15 \% \text {; a distorção idade- } \\
\text { série no ensino fundamental }-10 \% \text {; os } \\
\text { docentes com ensino superior no ensino } \\
\text { fundamental - } 15 \% \text {; a média de horas aula } \\
\text { diárias no ensino fundamental - } 15 \% \text {; e o } \\
\text { resultado do IDEB no ensino fundamental } \\
-25 \% \text {. A fórmula é apresentada abaixo } \\
\text { conforme Equação } 4 \text {. }\end{array}$ & $\begin{array}{l}\text { Entende-se que quanto maior a } \\
\text { eficácia orçamentária, maior será o } \\
\text { nível de escolaridade nos } \\
\text { municípios, conforme sustentam os } \\
\text { estudos de Queiroz et al. (2013), } \\
\text { Neves et al. (2015), Costa (2012) e } \\
\text { Wright e Paulo (2014). }\end{array}$ \\
\hline Saúde & SAUDE & $\begin{array}{l}\text { Para essa variável foi utilizado como proxy } \\
\text { o índice FIRJAN, visto conter os anos a } \\
\text { que este estudo se propõe. A nota para } \\
\text { esse índice é atribuída da seguinte forma: } \\
\text { utilizando o número de consultas pré-natal, } \\
\text { os óbitos obtidos por causas mal-definidas, } \\
\text { os óbitos infantis por causas evitáveis e a } \\
\text { internação sensível à atenção básica. Foi } \\
\text { atribuído peso de } 25 \% \text { em cada conceito. } \\
\text { A fórmula é apresentada abaixo conforme } \\
\text { Equação } 5 \text {. }\end{array}$ & $\begin{array}{l}\text { É esperado que quanto maior a } \\
\text { eficácia orçamentária, maior será o } \\
\text { nível de escolaridade, conforme } \\
\text { sustentam os estudos de Queiroz et } \\
\text { al. (2013), Neves et al. (2015) e } \\
\text { Oliveira e Passador (2014). }\end{array}$ \\
\hline
\end{tabular}

Nota. Fonte: Elaboração própria (2016).

Grau de Eficiência Orçamentária (GEO) - é a variável dependente da presente pesquisa, representada por uma proxy medida segundo a Equação apresentada a seguir, de 
acordo com Santana et al. (2007). Para a utilização do valor do GEO foi considerado o módulo do resultado.

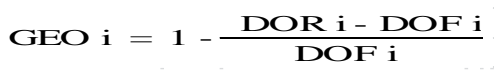

Em que:

GEO $\mathrm{i}=$ Grau de eficácia orçamentária;

$\mathrm{DOR}_{\mathrm{i}}=$ Despesas orçamentária realizadas;

DOF $_{\mathrm{i}}=$ Despesas orçamentária fixadas.

Emprego e renda (EmpRenda) - essa variável tem como fonte o Ministério do Trabalho e Emprego. São utilizadas duas dimensões: o Emprego, que analisa a geração de emprego e a capacidade de absorção de mão de obra; e a Renda, que avalia a geração de renda e sua distribuição no mercado de trabalho do município. Cada uma das duas dimensões representa 50\% do índice de Emprego\&Renda, cujo objetivo é captar a situação econômica como característica da estrutura do mercado de trabalho dos municípios. Assim, essa variável é medida pela seguinte Equação:

$$
\begin{aligned}
& \text { EmpRenda } a_{i t}=\gamma \text { Geração de Emprego }+ \\
& +\gamma \text { Capacidade de absorção da Mão }- \text { de }- \text { Obra } \\
& +\gamma \text { Geração de Renda } \\
& +\gamma \text { Distribuição no mercado de trabalho }
\end{aligned}
$$

Índice FIRJAN de desenvolvimento municipal da educação (IFDM - Educação) essa variável leva em consideração seis indicadores, conforme descritos no quadro 1. Esse índice serve para captar a oferta de educação infantil, como, também, a qualidade da educação prestada no ensino fundamental, em escolas públicas e privadas. Ao ensino fundamental foi atribuído peso de $80 \%$, distribuído entre cinco indicadores: $55 \%$ para indicadores-meio e $25 \%$ para indicadores-fim. Já o ensino infantil ficou com os $20 \%$ restantes do IFDM - Educação. Assim, essa variável é medida pela seguinte Equação:

$$
\begin{gathered}
E_{D U} C_{i t}=\gamma \text { Matrículas na educação infantil }+ \\
+\gamma \text { Abandono no ensino fundamental } \\
+\gamma \text { Distorção idade }- \text { série no ensino fundamental } \\
+\gamma \text { Docentes com ensino superior no ensino fundamental } \\
+\gamma \text { Média de horas aula diárias no ensino fundamental } \\
+\gamma \text { Resultado do IDEB no ensino fundamental }
\end{gathered}
$$

Índice FIRJAN de desenvolvimento municipal da saúde (IFDM - Saúde) - essa variável tem foco na saúde básica. Contempla quatro indicadores, que têm peso de $25 \%$ cada, conforme é descrito na tabela 1. Assim, essa variável é medida pela seguinte Equação:

$$
\begin{aligned}
& \text { SAUDE } E_{i t}=\gamma \text { Número de consultas pré }- \text { natal }+ \\
& +\gamma \text { Óbitos obtidos por causas mal }- \text { definidas } \\
& +\gamma \text { Óbitos infantis por causas evitáveis } \\
& \quad+\gamma \text { Internação sensível à atenção básica }
\end{aligned}
$$

É importante destacar que a metodologia do índice FIRJAN de emprego e renda, saúde e educação permite encontrar de forma precisa se a melhoria de um município é decorrente de boas práticas de políticas públicas implementadas pelo próprio município ou se aconteceu em virtude da queda dos demais municípios.

Esse índice varia de 0 (mínimo) a 1 ponto (máximo). O quanto mais próximo de 1 maior o desenvolvimento da localidade, conforme apresentado na Tabela 2: 
Tabela 2

Parâmetros para interpretação do IFDM

\begin{tabular}{c|c}
\hline VARIAÇÁO DO ÍNDICE & CLASSIFICAÇÃO \\
\hline De 0 a 0,4 & Baixo desenvolvimento \\
\hline De 0,4 a 0,6 & Regular desenvolvimento \\
\hline De 0,6 a 0,8 & Moderado desenvolvimento \\
\hline De 0,8 a 1 & Alto desenvolvimento \\
\hline
\end{tabular}

Nota. Fonte: Portal do Índice FIRJAN (2015).

\section{RESULTADOS}

Conforme definido na metodologia, o universo da pesquisa consiste dos 223 municípios do Estado da Paraíba. Desses, 14 não apresentaram todas as informações em anos diferenciados. Em virtude disso, esses municípios, que não apresentaram os dados completos em todos os anos e para todas as variáveis, foram retirados da amostra e, assim, permaneceram os 209 municípios no estudo.

Utilizou-se a análise de dados em painel balanceado com efeitos fixos, visto que, ao contrário do pooled, considera a heterogeneidade dos municípios que podem influenciar a variável dependente do estudo, isto é, leva em consideração as especificidades dos municípios ao longo do tempo, ou seja, o impacto das variáveis no período em análise. Evita-se, assim, os problemas da omissão de variáveis, o que leva, portanto, a estimadores mais consistentes e eficientes.

A variável HABM apresenta outliers, visto que existem municípios com grande número populacional e outros com poucos habitantes. Mostra a discrepância entre os municípios da Paraíba. Por apresentar uma disparidade grande na variável HABM, mostrando uma distribuição anormal, aplicou-se o logaritmo natural para essa variável, agora denominada InHABM. Também se decidiu por aplicar o logaritmo natural na variável SAUDE, pois se constatou a importância de melhor adequar as variáveis ao modelo.

Apesar do número de observações ser alto, 1.881 observações, e isso já pressupor a normalidade dos dados, foram feitos os testes de normalidade de Shapiro-Wilk e ShapiroFrancia. Constatou-se que apenas a variável EDUC apresenta normalidade nos dados, conforme Tabela 3, abaixo.

Tabela 3

Testes de Normalidade de Shapiro-Wilk e Shapiro-Francia nos períodos de 2005 a 2013

\begin{tabular}{l|r|r}
\hline \multicolumn{1}{c|}{ Variável } & \multicolumn{1}{c|}{ Shapiro-Wilk } & \multicolumn{1}{c}{ Shapiro-Francia } \\
\hline GEO & 0,00014 & 0,00022 \\
\hline InHABM & 0,00000 & 0,00001 \\
\hline EDUC & 0,17773 & 0,25202 \\
\hline EmpRenda & 0,00000 & 0,00001 \\
\hline InSAUDE & 0,00000 & 0,00001 \\
\hline
\end{tabular}

Nota. Fonte: Elaboração própria (2016) com os dados extraídos de SAGRES, IBGE e FIRJAN.

Em seguida, foi realizada a estatística descritiva das variáveis, descritas na Tabela 4. A variável GEO, conforme modelo apresentado, é a variável dependente, explicada pelas demais variáveis independentes: EDUC, EmpRenda, InSAUDE e InHABM como uma variável de controle.

Tabela 4

Estatística descritiva das variáveis do modelo nos períodos de 2005 a 2013

\begin{tabular}{l|r|r|r|r|r}
\hline \multicolumn{1}{c|}{ Estatística } & \multicolumn{1}{c|}{ GEO } & \multicolumn{1}{c|}{ InHABM } & \multicolumn{1}{c}{ EDUC } & \multicolumn{1}{c}{ EmpRenda } & \multicolumn{1}{c}{ InSAUDE } \\
\hline Média & 1,127092 & 9,051869 & 0,5415434 & 0,4027247 & $-0,5692249$ \\
\hline Desvio-Padrão & 0,1935049 & 0,9167358 & 0,1005181 & 0,0873441 & 0,277974 \\
\hline Observações & 1.881 & 1.881 & 1.881 & 1.881 & 1.881 \\
\hline
\end{tabular}


Tabela 4 (continuação)

\begin{tabular}{l|r|r|r|r|r}
\hline \multicolumn{1}{c|}{ Estatística } & \multicolumn{1}{c|}{ GEO } & \multicolumn{1}{c|}{ InHABM } & \multicolumn{1}{c|}{ EDUC } & \multicolumn{1}{c}{ EmpRenda } & \multicolumn{1}{c}{ InSAUDE } \\
\hline Mínimo & 0,3941116 & 7,24065 & 0,246868 & 0,1439972 & $-2,59083$ \\
\hline Máximo & 1,713902 & 13,55367 & 0,8817995 & 0,8827442 & $-0,1046459$ \\
\hline Assimetria & $-0,0687741$ & 1,210343 & 0,0371865 & 1,123141 & $-1,111657$ \\
\hline Curtose & 3,138443 & 6,068068 & 2,911983 & 7,023269 & 5,381835 \\
\hline
\end{tabular}

Nota. Fonte: Elaboração própria (2016) com os dados extraídos de SAGRES, IBGE e FIRJAN.

A variável GEO apresenta média de 1,12, ultrapassando o valor de 1. Constata-se que os municípios da Paraíba, em geral, utilizam menos recursos que o programado na LOA. Analisando o mínimo $(0,39)$ e o máximo $(1,71)$ do GEO, observa-se que os municípios paraibanos que apresentam valores menores que 1 fazem uso de mais recursos que os previamente fixados, ou seja, utilizam mais créditos adicionais. Comprometem a eficácia orçamentária. Em contrapartida, municípios com valores acima de 1 tendem a ser menos eficazes, pois não utilizam os recursos mínimos previamente estabelecidos.

A variável GEO, por apresentar curtose de 3,13 , ou seja, um valor próximo de 3 , identifica que os municípios paraibanos, em média, apresentam normalidade dos dados. A variável EDUC, em análise da curtose, demonstra quase o mesmo comportamento que a GEO. Já as variáveis EmpRenda e InSAUDE apresentam uma distribuição fora da normalidade. Identificam diferenças entre a renda e a saúde nos municípios paraibanos, como também a variável InHABM, que mostra a diferença de quantidade de habitantes nos municípios paraibanos, o que pode comprometer a eficácia orçamentária.

Logo após a análise descritiva, foram realizados testes de correlações descritos na Tabela 5, que mostra as correlações de Pearson e Spearman entre as variáveis do modelo e suas respectivas significâncias. Os valores acima da diagonal 1.0000 são os referentes à correlação de Pearson e os valores abaixo dessa diagonal são os da correlação de Spearman para as variáveis da pesquisa.

Tabela 5

Correlação de Pearson e Spearman para as variáveis do estudo no período de 2005 a 2013

\begin{tabular}{|c|c|c|c|c|c|}
\hline & GEO & InHABM & EDUC & EmpRenda & InSAUDE \\
\hline \multirow[t]{2}{*}{ GEO } & 1.0000 & $-0.1472^{* * *}$ & $0.3216^{* * *}$ & -0.0087 & $0.1992^{* * *}$ \\
\hline & & 0.0000 & 0.0000 & 0.7066 & 0.0000 \\
\hline \multirow[t]{2}{*}{ InHABM } & $-0.1444^{\star \star \star}$ & 1.0000 & $-0.0582^{* * *}$ & $0.3559^{* * *}$ & 0.0139 \\
\hline & 0.0000 & & 0.0116 & 0.0000 & 0.5480 \\
\hline \multirow[t]{2}{*}{ EDUC } & $0.3195^{* * *}$ & $-0.0794^{* * *}$ & 1.0000 & $0.0450^{* *}$ & $0.4422^{* * *}$ \\
\hline & 0.0000 & 0.0000 & & 0.0511 & 0.0000 \\
\hline \multirow[t]{2}{*}{ EmpRenda } & -0.0085 & $0.1657^{* * *}$ & $0.0421^{* *}$ & 1.0000 & $0.1798^{* * *}$ \\
\hline & 0.7136 & 0.0000 & 0.0680 & & 0.0000 \\
\hline \multirow[t]{2}{*}{ InSAUDE } & $0.2057^{* * *}$ & $-0.0372^{*}$ & $0.4554^{* * *}$ & $0.1471^{* * *}$ & 1.0000 \\
\hline & 0.0000 & 0.1069 & 0.0000 & 0.0000 & \\
\hline
\end{tabular}

Nota. Fonte: Elaboração própria (2016), com os dados extraídos de SAGRES, IBGE e FIRJAN. Legenda: Significância ${ }^{*}=10 \% ;{ }^{* *}=5 \% ;{ }^{* * *}=1 \%$.

Pela correlação de Pearson nota-se que quase todas as variáveis do modelo, InHABM, EDUC e SAUDE apresentaram forte significância, a nível de 1\%, com a dependente GEO. 
Apenas a variável EmpRenda não obteve qualquer significância com a GEO. De igual modo, quando observados na Tabela 5 , os valores obtidos pela correlação de Spearman, percebe-se que a única variável que não apresentou significância com a GEO foi a EmpRenda.

Observa-se na Tabela 5 que a variável InHABM, apesar da significância, apresenta valor de correlação negativo com a variável GEO. Indica que municípios com grande contingente populacional apresentam menor grau de eficácia orçamentária. Isso faz com que a hipótese 2, que afirma que os municípios maiores apresentam maior grau de eficácia orçamentária comparados a municípios menores, não seja aceita, já que GEO e InHABM são inversamente proporcionais. Isso diverge dos estudos de Poker et al. (2013) e Neves et al. (2015) e corrobora os estudos de Oliveira e Passador (2014) e Queiroz et al. (2013).

Ademais, é importante destacar que municípios menores parecem não dispor da mesma eficácia no planejamento e incorporação de novas práticas que os municípios maiores, inclusive apresentam dificuldades para elaboração adequada do orçamento (Santana et al., 2007).

A Tabela 5 mostra, também, uma relação significativa e negativa entre as variáveis EDUC e InHABM. Demonstra que os municípios que apresentam maior contingente populacional, possuem menores indicadores educacionais. Isso não se repete quando se observa a variável EmpRend, pois a mesma apresenta melhores resultados em municípios que têm população maior.

Analisando-se as variáveis EDUC e InSAUDE, que apresentaram significância ao nível de $1 \%$ com a GEO, além de correlação positiva, depreende-se que os indicadores sociais de educação e saúde influenciam positivamente a eficácia orçamentária, não rejeitando, assim, a hipótese número 1. Porém, a variável EmpRenda, além de não ter significância com a GEO, a correlação é negativa, evidencia que os municípios paraibanos apresentam rendas variadas e que quanto maior o índice de emprego e renda, menor será o grau de eficácia orçamentária.

Ainda, com relação às correlações entre as variáveis, cabe destacar que EDUC e EmpRend expressam correlações significativas e positivas com a variável InSAUDE. Revelam que municípios que apresentam bons indicadores de educação e emprego e renda apresentam melhores resultados no quesito saúde.

Para a escolha dos dados em painel de efeitos fixos foi realizado o teste de Hausman, apresentado na Tabela 6, que considera que as características invariantes no decorrer do tempo são únicas para cada indivíduo, ou seja, que não se relacionam com as características de outros indivíduos. No caso do presente estudo, isso mostra que cada município possui características próprias e que isso não depende dos outros municípios. Então, a estimação capta a heterogeneidade dos municípios no que é constante, ou seja, a diferença de município para município que não varia no decorrer do tempo, como alguns fatores ambientais que tendem a ser invariantes. Como observado na Tabela 6, o resultado da prob>chi2 foi menor que $5 \%$. Constata-se que trabalhar com painel com efeitos fixos é mais apropriado para este estudo.

Tabela 6

Teste de Hausman para os períodos de 2005 a 2013

\begin{tabular}{l|c|c|c|c}
\hline \multicolumn{1}{c|}{ Estatística } & Efeitos fixos & Efeitos aleatórios & Diferença & $\begin{array}{c}\text { Erro padrão da } \\
\text { diferença }\end{array}$ \\
\hline EDUC & 0,7683925 & 0,6965754 & 0,0718172 & 0,0244958 \\
\hline EmpRenda & 0,090302 & 0,0461549 & 0,0441471 & 0,0310782 \\
\hline InSAUDE & 0,0537956 & 0,0560105 & $-0,0022149$ & 0,0096662 \\
\hline InHABM & $-0,0099448$ & $-0,0185237$ & 0,0085789 & 0,0038336 \\
\hline \multicolumn{2}{r|c}{ Prob>chi2 $=0.0000$} \\
\hline
\end{tabular}

Nota. Fonte: Elaboração própria (2016), com nos dados extraídos de SAGRES, IBGE e FIRJAN.

Para dar maior robustez para a escolha dos dados em painel de efeitos fixos, foi realizado o teste de Chow. Constatou-se que os efeitos fixos são os mais apropriados para o modelo em estudo, conforme é apresentado na Tabela 7 , em que o Prob $>F=0,0000$, ou seja, menor que $5 \%$.

Após a verificação pelo teste de Hausman do melhor modelo a ser trabalhado, para dar maior robustez para a escolha dos dados em painel de efeitos fixos, foi realizado o teste de 
Chow. Constatou-se que os efeitos fixos são os mais apropriados para o modelo em estudo, conforme é apresentado na Tabela 7 , em que o Prob $>F=0,0000$, ou seja, menor que $5 \%$.

Seguiu-se com a análise da aplicação do modelo empírico, apresentado na Equação 1 dos procedimentos metodológicos. A Tabela 7 mostra os resultados da regressão do modelo.

Tabela 7

Coeficientes e estatísticas obtidas a partir do modelo da regressão

\begin{tabular}{|c|c|c|c|c|c|c|}
\hline GEO & Coeficiente & $\begin{array}{l}\text { Desvio- } \\
\text { padrão }\end{array}$ & $t$ & $p$-value & 95\% Confiança & Intervalo \\
\hline EDUC & 0,7683925 & 0,0560911 & 13,70 & 0,000 & 0,6583763 & 0,8784088 \\
\hline EmpRenda & 0,090302 & 0,064895 & 1,39 & 0,164 & $-0,0369823$ & 0,2175863 \\
\hline InSAUDE & 0,0537956 & 0,0211523 & 2,54 & 0,011 & 0,0123078 & 0,0952833 \\
\hline InHABM & $-0,0099448$ & 0,0069263 & $-1,44$ & 0,151 & $-0,02353$ & 0,0036404 \\
\hline _cons & 0,7952478 & 0,0777055 & 10,23 & 0,000 & 0,6428373 & 0,9476583 \\
\hline$F(208,1668)$ & 5,13 & & & \multicolumn{3}{|c|}{ Prob>F $\quad 0,0000$} \\
\hline sigma_u & 0,11724016 & sigma_e & 0,1500173 & \multicolumn{3}{|c|}{ rho } \\
\hline
\end{tabular}

Nota. Fonte: Elaboração própria (2016) com os dados extraídos de SAGRES, IBGE e FIRJAN.

Analisando a regressão, apresentada na Tabela 7 , verifica-se que as variáveis EmpRenda e InHABM não foram significantes. Todavia, as demais variáveis foram significantes. logo, explicam o comportamento da variável dependente e confirmam a hipótese 1 de que os indicadores socioeconômicos influenciam positivamente a eficácia orçamentária municipal, confirmando igualmente os achados dos estudos de Neves et al. (2015) e Wright e Paulo (2014).

A variável de controle InHABM, que representa a quantidade de habitantes, apresenta relação inversa com a variável dependente GEO observando-se o sinal negativo do coeficiente, indicando que a quantidade de habitantes de determinado município influencia negativamente o grau de eficácia orçamentária, ou seja, municípios mais populosos apresentam GEO menores.

Além disso, percebe-se que as variáveis EDUC e InSAUDE são significantes ao nível de $1 \%$ e $5 \%$, respectivamente, com a GEO e apresentam correlações positivas. Isso conduz a inferência de que bons indicadores de educação e saúde fornecem elevados graus de eficácia orçamentária. Portanto, a eficácia orçamentária é refletida nesses indicadores sociais.

\section{CONCLUSÃO}

Este estudo buscou verificar se o grau de eficácia orçamentária é influenciado pelos indicadores socioeconômicos, adotando-se como unidades de análise os municípios da Paraíba. Para isso, foram utilizados como proxies para educação, renda e saúde as notas atribuídas pelo índice FIRJAN no período de 2005 a 2013. Além disso, o tamanho da população também foi considerado importante para se medir o grau de eficácia orçamentária.

Os resultados obtidos revelaram que a maioria dos municípios apresenta bom grau de eficácia, ou seja, GEO entre 0,9 e 1,1. Como o GEO apresenta média de 1,12, deduz-se que os municípios utilizam menos recursos que o ideal.

Referente à correlação entre as variáveis, percebe-se que as de saúde e educação apresentam forte correlação com a GEO. Indicam que os gastos nesses dois segmentos exigem mais despesas públicas. Além disso, o número de habitantes também apresentou correlação com a GEO, contudo negativa. Constata-se que a quantidade da população influencia de forma inversa o grau de eficácia orçamentária. Já a variável EmpRenda não apresenta significância e além disso mostra correlação negativa. Conclui-se, pois, que municípios com maior emprego e renda têm menor grau de eficácia orçamentária.

Os resultados da regressão mostram a não significância das variáveis EmpRenda e InHABM com a GEO, enquanto que as demais variáveis foram significantes. Portanto, confirmou-se a hipótese de que a eficácia orçamentária é influenciada positivamente pelos 
indicadores socioeconômicos, corroborando os trabalhos de Neves et al. (2015) e Wright e Paulo (2014).

Porém, a hipótese 2, que afirma que os municípios maiores apresentam maior grau de eficácia orçamentária comparado a municípios menores, foi rejeitada, pois a variável InHABM, além de não ser significante com a GEO, seu coeficiente é negativo, confirmando o estudo de Queiroz et al. (2013), ao encontrarem pouca relação entre tamanho da população e grau de evidenciação, mais precisamente, transparência pública.

Sugere-se para futuras pesquisas a utilização de outras proxies para as variáveis propostas, como também o uso de outras unidades de análise. Além disso, outra forma de medir o grau de eficácia orçamentária pode refletir os resultados obtidos.

\section{REFERÊNCIAS}

Aristigueta, M. P., Cooksy, L. J., \& Nelson, C. W (2001). The role of social indicators in developing a managing for results system. Public Performance \& Management Review, 24(3), 254-269.

Bandeira-de-melo, R., Marcon, R., \& Alberton, A (2005). Teoria instrumental dos stakeholders em ambientes turbulentos: uma verificação empírica utilizando as doações políticas e sociais. Anais do Encontro Nacional da ANPAD, Brasília, DF, Brasil, 29.

Bárbara, S., \& Rodrigues, J. C. C (2005). De número a instrumento: o novo papel dos indicadores socioeconômicos na formulação de políticas públicas - um estudo de caso sobre a habitação em terra dos royalties do petróleo. Anais do Encontro Nacional da Associação Nacional de Pós-Graduação e Pesquisa em Planejamento Urbano e Regional - ANPUR, Salvador, BA, Brasil.

Bezerra, J. E., Filho (2013). Orçamento aplicado ao setor público: abordagem simples e objetiva (2a ed.). São Paulo: Atlas.

Boaventura, J. M. G., Cardoso, F. R., Silva, E. S., \& Silva, R. S. (2009). Teoria dos Stakeholders e Teoria da Firma: um estudo sobre a hierarquização das funções-objetivo em empresas brasileiras. Revista Brasileira de Gestão de Negócios, 11(32), 289-307.

Borges, E. F., \& Pereira, J. M. (2014). Educação fiscal e eficiência pública: um estudo das suas relações a partir da gestão de recursos municipais. Revista de Educação e Pesquisa em Contabilidade - REPeC. Brasília, 8(4), 437-453.

Constituição da República Federativa do Brasil. (1988). Recuperado em 15 de outubro, 2015, de: http://www.planalto.gov.br/ccivil_03/constituicao/Constituicaocompilado.htm

Carvalho, D (2007). Orçamento e contabilidade pública: teoria, prática e mais de 700 exercícios (3a ed.). Elsevier: Rio de Janeiro.

Castro, R. B. (2006). Eficácia, eficiência e efetividade na Administração Pública. Anais do Encontro da ANPAD. Salvador, BA, Brasil, 30.

Controladoria Geral da União (2009). Manual de controle interno. Brasília.

Decreto-Lei n. 200, de 25 de fevereiro de 1967 (1967). Dispõe sôbre a organização da Administração Federal, estabelece diretrizes para a Reforma Administrativa e dá outras providências. Recuperado em 14 de novembro, 2017, de http://www.planalto.gov.br/ccivil_03/decreto-lei/del0200.htm

Donaldson, T., \& Preston, L. E. (1995). The stakeholder theory of the corporation: Concepts, evidence, and implications. Academy of management Review, 20(1), 65-91.

Federação das indústrias do estado do Rio de Janeiro. Índice Firjan de Desenvolvimento Municipal. IFDM. Recuperado em 6 janeiro, 2016, de http://www.firjan.com.br/ifdm/

Freeman, R. E. (2010). Strategic management: a stakeholder approach. University of Minnesota. Cambridge University Press.

Giacomoni, J. (2012). Orçamento Público (16a ed.). São Paulo: Atlas. 
Gomes, R. C. (2006). Stakeholder management in the local government decision-making area: evidences from a triangulation study with the English local government. BAR-Brazilian Administration Review, 3(1), 46-63.

Instituto Brasileiro de Geografia e Pesquisa. Recuperado em 23 novembro, 2015, de http://www.ibge.gov.br/home/

Jannuzzi, P. M. (2002). Indicadores sociais na formulação e avaliação de políticas públicas. Revista Brasileira de Administração Pública, 36(1), 51-72.

Jannuzzi, P. M. (2012). Indicadores socioeconômicos na gestão pública. (2a ed. reimp.). Florianópolis: Departamento de Ciências da Administração: UFSC.

Khan, A., \& Hildrth, W. B. (2002) Budget theory in thepublic sector. Quorum Books.

Kashiwakura, H. K. (1997). A contabilidade gerencial aplicada ao orçamento-programa como instrumento de avaliação de desempenho. Monografia, Ministério da Fazenda, ESAF, Rio de Janeiro, Brasil.

Koscianski, R. (2003). O orçamento-programa como instrumento de planejamento e gerenciamento públicos. Tese de doutorado, Universidade Federal de Santa Catarina, SC, Brasil.

Lei n. 101, de 04 de maio de 2000 (2000). Estabelece normas de finanças públicas voltadas para a responsabilidade na gestão fiscal e dá outras providências. Recuperado em 14 de novembro, 2017, de http://www.planalto.gov.br/ccivil_03/leis/LCP/Lcp101.htm

Lei n. 4.320, de 17 de março de 1964 (1964). Estatui normas gerais de direito financeiro para elaboração e controle dos orçamentos e balanços da União, dos Estados, dos Municípios e do Distrito Federal. Recuperado em 15 de outubro, 2015, de http://www.planalto.gov.br/ccivil_03/LEIS/L4320.htm

Lima, S. C., \& Diniz, J. A. (2016). Contabilidade Pública: análise financeira governamental. São Paulo: Atlas.

Litpvsky, A., \& Macgillivray, A. (2007).Development as accountability: accountability innovators in action. United Kingdom.

Lück, H. (2000). Perspectivas da gestão escolar e implicações quanto à formação de seus gestores. Em Aberto, 17(72), 11-33.

Mendes, M., Miranda, R. B., \& Cossio, F. B. (2008). O fundo de participação dos municípios precisa mudar. Senado Federal, Brasil.

Moldau, J. H. (1998). Os fundamentos microeconômicos dos indicadores de desenvolvimento econômico. Revista de Economia Política, 18(3).

Neves, A. C., Diniz, J. A., \& Martins, V. G. (2015). Determinantes socioeconômicos da transparência fiscal. Anais do Congresso USP Controladoria e Contabilidade, São Paulo, SP, 15.

Nogueira, M. A. (2004). Um estado para a sociedade civil: temas éticos e políticos da gestão democrática. São Paulo: Cortez Editora.

Oliveira, L. R., \& Passador, C. S. (2014). Saúde pública no Brasil: a utilização do índice de desempenho do SUS na avaliação da alocação dos recursos dos municípios. Revista Eletrônica Gestão \& Saúde, 5(4), 2387-2405.

Poker, J. H., Jr., Nunes, R. C., \& Nunes, S. P. P. (2013). Uma avaliação de efetividade e eficiência do gasto em educação em municípios brasileiros. Cad. Fin. Públ., (13) 263-287.

Queiroz, D. B., Nobre, F. C., Silva, W. V., \& Araújo, A. O. (2013). Transparência dos municípios do Rio Grande do Norte: avaliação da relação entre o nível de disclosure, tamanho e características socioeconômicas. Revista Evidenciação Contábil \& Finanças-RECFin, 1(2), 38-51. 
Carla Janaina Ferreira Nobre, Josedilton Alves Diniz, Severino Cesário de Lima, Ronaldo José Rêgo de Araújo

Santana, E. W. F., Pessoa, L. G. de S. B., Cabral, L. M. M. do A. C., Santos, S. R. B. dos, \& Diniz, J. A. (2007). Eficácia orçamentária municipal: os impactos produzidos pela lei de responsabilidade fiscal. Anais do Encontro da Associação Nacional de Pós-Graduação e Pesquisa em Administração, Rio de Janeiro, RJ.

Santos, F. R. (2008). O emprego da análise de stakeholders em um plano estratégico para a gestão da mobilidade sustentável: estudo de caso do campus da universidade de Brasília. Dissertação de mestrado, Universidade de Brasília, Brasília, DF.

Varela, P. S. (2004). Indicadores sociais no processo orçamentário do setor público municipal de saúde: um estudo de caso. Dissertação de mestrado, Universidade de São Paulo, São Paulo, SP, Brasil.

Wright, G. A., \& Paulo, E. (2014). Análise dos fatores determinantes da transparência fiscal ativa nos municípios brasileiros. Anais do Congresso Anpcont, Rio de Janeiro, Brasil, 8. 